\section{Biotinidaseaktivitätsmessung aus Trockenblut}

G. F. Hoffmann ${ }^{1}$, C.-D. Langhans ${ }^{2}$ und A. Schulze ${ }^{3}$

${ }^{1}$ Universitätsklinikum Heidelberg, Zentrum für Kinder- und

Jugendmedizin, Heidelberg, Deutschland

${ }^{2}$ Universitätsklinik für Kinder- und Jugendmedizin, Stoffwechsellabor - GCMS, Heidelberg, Deutschland ${ }^{3}$ Clinical and Metabolic Genetics The Hospital for Sick Children, University of Toronto, Toronto, Kanada

Englischer Begriff biotinidase activity in dried blood spot (DBS) specimen

Definition Bestimmung der Aktivität der Biotinidase im Trockenblut von Neugeborenen zum Screening auf das Vorliegen eines Biotinidasemangels.

Physikalisch-chemisches Prinzip Die Biotinidase aus der Trockenblutprobe setzt $p$-Aminobenzoesäure aus Biotinylp-Aminobenzoesäure frei. Die Konzentration der gebildeten p-Aminobenzoesäure wird durch Messung der Lichtabsorption nach Bildung eines Diazofarbstoffes als Endpunktmethode mit Substratüberschuss spektrophotometrisch bestimmt.

Einsatzgebiet Neugeborenenscreening.

Untersuchungsmaterial Vollblut getrocknet auf Filterpapier (= Trockenblut).
Instrumentierung Mikrotiterplattenphotometer, Mikrotiterplattenzentrifuge, Mikrotiterfilterplatten, Mikrotiterplatten, Multipuncher oder Handstanzen, 12-Kanal-Pipette.

Spezifität Diagnostische Spezifität im Screening: $>99,9 \%$.

Sensitivität Diagnostische Sensitivität im Screening: >99\%.

Fehlermöglichkeit Falsch positives Screening: hohe Temperatur; falsch negatives Screening: Bluttransfusion, Sulfonamid-Therapie.

Praktikabilität - Automatisierung - Kosten Praktikabilität: sehr gut.

Kosten: ca. 0,50 Euro/Test (Chemikalien und Verbrauchsmaterialien).

Bewertung - Methodenhierarchie (allg.) Die photometrische Bestimmung der Biotinidaseaktivität aus Trockenblut stellt ein zuverlässiges Verfahren zum Ausschluss eines Biotinidasemangels im Neugeborenenscreening dar.

\section{Literatur}

Zabransky S (2001) Biotinidasemangel. In: Zabransky S (Hrsg) Screening auf angeborene endokrine und metabole Störungen. Springer, Wien/New York, S 250-254 